\title{
Programas de intervenção nas escolas brasileiras: uma contribuição da escola para a educação em saúde
}

\author{
Intervention programs in Brazilian schools: a contribution of school to health education \\ Ahécio Kleber Araújo Brito', Francisca Islandia Cardoso da Silva², Nanci Maria de França ${ }^{3}$
}

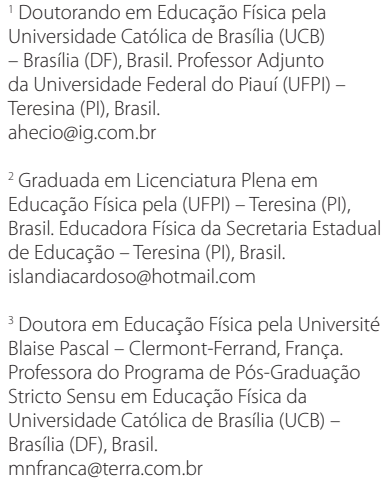

${ }^{2}$ Graduada em Licenciatura Plena em Educação Física pela (UFPI) - Teresina (PI), Brasil. Educadora Física da Secretaria Estadual de Educação - Teresina (PI), Brasil. islandiacardoso@hotmail.com

${ }^{3}$ Doutora em Educação Física pela Université Blaise Pascal - Clermont-Ferrand, França. Professora do Programa de Pós-Graduação Stricto Sensu em Educação Física da Universidade Católica de Brasília (UCB) Brasília (DF), Brasil.

mnfranca@terra.com.br

RESUMO Artigo com objetivo de revisar programas de intervenção direcionados à saúde realizados nas escolas brasileiras. Pesquisaram-se dados dos bancos Lilacs, SciELO e Medline, dissertações, teses e documentos oficiais. Foram encontrados dez programas de saúde e um número reduzido de intervenções em atividade física nas escolas. Nos núcleos de apoio à saúde da família, identificou-se pequena participação dos profissionais de educação física. Concluiu-se que os programas de intervenção realizados nas escolas estão reduzindo o sedentarismo e integrando ações de educação à saúde; e que, nos programas de apoio à saúde da família, faz-se necessária maior participação dos profissionais de educação física.

PALAVRAS CHAVE: Educação; Promoção de saúde; Escola.

\begin{abstract}
Article aiming to revise intervention programs targeted to health conducted in Brazilian schools. It was researched data banks Lilacs, SciELO and Medline, dissertations, theses and official documents. Ten programs of health and a reduced number of interventions were found in physical activity in schools. In the nuclei of supporting family health was identified weak participation of physical education professionals. It was concluded that intervention programs conducted in schools are reducing physical inactivity and integrating health education activities; and for programs that support family health it is necessary greater participation of physical education professionals.
\end{abstract}

KEYWORDS: Education; Health promotion; School. 


\section{Introdução}

O ambiente escolar é o contexto ideal para o desenvolvimento de práticas promotoras de saúde, já que exerce influência na aquisição de valores e estimula o exercício da cidadania. Com base no pressuposto - segundo o qual, por meio da educação em saúde, poder-se-ia estimular comportamentos, valores e atitudes entre os indivíduos -, é necessário que as estratégias com tal fim se façam de modo a contemplar a individualidade e o contexto social dos indivíduos, recorrendo a estratégias pedagógicas, sociais e psicológicas para aumentar suas chances de sucesso (GONÇALVES et al, 2008; PIRES; MUSSI, 2008; SANTOS; CAETANO; MOREIRA, 2011).

$\mathrm{Na}$ área da saúde, os profissionais utilizam-se da educação em saúde como um instrumento de trabalho na construção da relação com os usuários dos serviços de saúde, na medida em que a saúde perpassa todos os aspectos do viver humano e requer, para a transformação dos sujeitos, uma profunda interação entre o profissional de saúde e a população, com vistas a permear as condutas que gerem saberes (SANTOS et al, 2011).

Deste modo, é consenso que as crianças e os adolescentes têm práticas de hábitos de vida cada vez mais errôneos, do ponto de vista da saúde, em decorrência das facilidades proporcionadas pelos avanços da tecnologia e do desenvolvimento industrial. Além disso, as crescentes modernização e urbanização das cidades, que têm reduzido os espaços públicos para o lazer e promovido o aumento da violência urbana, têm modificado o comportamento dos jovens, substituindo gradativamente a prática de um lazer ativo - brincar e jogar nas ruas e praças - pela de um lazer passivo - jogar no computador ou assistir a televisão -, esta associada à ingestão de alimentos hipercalóricos. Tais mudanças são consideradas favorecedoras do desenvolvimento das doenças crônicas não transmissíveis (WORLD HEALTH ORGANIZATION, 2000).

O sedentarismo pode ser considerado uma epidemia mundial, pois compromete cerca de $70 \%$ da população do planeta. É fator de risco para doenças crônicas não transmissíveis, como diabetes tipo 2 , hipertensão, hipercolesterolêmica, obesidade, doenças cardiovasculares, osteoporose e algumas formas de câncer. Estimativas econômicas de vários países consideram o sedentarismo responsável por 2 a $6 \%$ dos custos totais em saúde pública. No Brasil, não há dados sobre o custo do sedentarismo, mas recente relatório elaborado pelo Banco Mundial atribuiu 66\% dos gastos em saúde às doenças crônicas não transmissíveis em todo o País (POZENA; CUNHA, 2009; VERGNAUD et al, 2008).

Por outro lado, a prática regular de atividade física apresenta uma relação inversa com o risco de doenças e tem um efeito positivo na qualidade de vida das pessoas, sendo consenso de vários autores que a saúde e a qualidade de vida do homem podem ser preservadas e aprimoradas pela prática regular de atividades físicas, sob a perspectiva da aptidáo física e da saúde (MOURA et al, 2012; REIS; REIS; HALLAL, 2011; TENDAIS et al, 2011).

As agências mundiais de saúde têm editado, nos últimos tempos, diversos documentos contendo estratégias globais para o incentivo às práticas saudáveis, objetivando a promoção da saúde e a redução das doenças não transmissíveis, sendo a alimentação saudável e a prática regular de atividades físicas constantemente recomendadas. Diante dessa discussão, a Organização Mundial da Saúde (OMS) sugere que os programas de exercícios físicos deveriam contemplar o aspecto lúdico, agradável, de forma que tais atividades se tornem mais atraentes, levando à formação desses hábitos saudáveis para toda a vida (WORLD HEALTH ORGANIZATION, 2004). Portanto, a escola é a principal (e talvez o mais acessível) veículo de formação para as crianças e adolescentes, e deve cumprir seu papel de educar para esses fins, uma vez que as famílias não possuem as informaçôes necessárias sobre esses aspectos.

Ante o exposto, o estudo pretende responder ao seguinte questionamento: quais são os programas de intervenção em saúde realizados nas escolas do Brasil? Ademais, não se pode falar em educação para a saúde sem políticas públicas de intervenção nas escolas, uma vez que educação e saúde se inter-relacionam mutuamente. Por essa razão, pode-se argumentar e justificar o presente ensaio, que tem como objetivo revisar os programas de intervenção direcionados a saúde realizados 
nas escolas brasileiras, e, assim, possibilitar a ingerência da escola como um veículo importante para a educação e a promoção da saúde dos estudantes.

\section{Métodos}

Este trabalho utilizou o método da revisão bibliográfica e, para esse fim, buscou periódicos científicos dos bancos de dados Lilacs, SciELO e Medline e dissertaçôes/teses pesquisadas em sites de busca da internet. Os critérios para citação do documento foram os seguintes: programa ou projeto com intervenção direcionada à educação ou à promoção da saúde na escola, com texto publicado em periódico indexado no portal qualis - CAPES (Coordenação de Aperfeiçoamento de Pessoal de Nível Superior). Foram pesquisados também: a) dissertaçóes de mestrado e teses de doutorado nos sites das universidades brasileiras que ofertam programas stricto sensu em áreas da saúde; b) dados fornecidos pela Organização Mundial de Saúde (OMS) e pelo Ministério da Saúde (MS). A coleta dos dados foi realizada durante o segundo semestre do ano de 2010, e os autores citados na revisão trazem experiências de intervenções em saúde realizadas nas escolas do Brasil, desde o ano 2000 até os dias atuais.

\section{Resultados}

Após a análise dos dados encontrados, verificou-se que dez programas atendem aos critérios de inclusão estabelecidos para a presente revisão. Dos dez programas citados, quatro receberam a denominação de projeto, quatro de programa e apenas dois foram denominados de estudo, talvez por terem sido originados de dissertaçóes de mestrado. Os programas e projetos apresentam, sobretudo, pesquisas com delineamento experimental; apenas um projeto possui característica de estudo transversal; e em dois programas não foi identificada pesquisa científica. Cabe também destacar que o Programa Saúde na Escola foi citado apenas uma vez por se tratar de um programa único, embora seja desenvolvido em diversos municípios brasileiros. Destaca-se, a seguir, cada projeto ou programa encontrado.

Inicia-se com o programa governamental Política Nacional de Promoção da Saúde (PNPS), posto que sua atuação ultrapassa as esferas de gestão do Sistema Único de Saúde, (SUS) interagindo com as demais políticas públicas e da sociedade. Aprovado em 2006, o principal objetivo do programa é Promover a qualidade de vida e reduzir a vulnerabilidade e os riscos à saúde relacionados aos seus determinantes e condicionantes - modos de viver, condições de trabalho, habitação, ambiente, educação, lazer, cultura, acesso a bens e serviços essenciais. À luz da promoção da atividade física, a Política Nacional de Promoção da Saúde (PNPS) propóe o compromisso da sociedade e de suas instituiçôes com a adoção de modos de vida mais saudáveis. A priorização do incentivo às práticas corporais na PNPS reconhece a relevância epidemiológica do tema sedentarismo. Os dados da Vigilância de Fatores de Risco e Proteção para Doenças Crônicas por Inquérito Telefônico (VIGITEL), pesquisa que faz parte das açóes da PNPS, indicaram, para o ano de 2011, que a prevalência de sobrepeso e obesidade entre adultos é de $48,55 \%$ e 15,85 , respectivamente; entre adolescentes, é de $47 \%$ e 14\% (BRASIL, 2010).

Chamou-nos a atenção também o Projeto de Avaliação do Estado Nutricional de Escolares (AENE), que teve origem na Tese de Doutorado: Avaliação do estado de nutrição de escolares do município de São Paulo: uma experiência multidisciplinar envolvendo professores de educação física do Programa de PósGraduação Interunidades de Nutrição Humana Aplicada da Universidade de São Paulo - USP. O trabalho realizou um curso de capacitação para professores de educação física da Secretária Municipal de Educação - SME. Em seguida, os mesmos avaliaram 9720 escolares de 10 a 18 anos, no ano de 2003, sendo 4829 meninas e 4826 meninos. Dentre os meninos, 11\% apresentaram desnutrição leve ou moderada e 14\% algum grau de obesidade; os índices entre meninas foram $12,7 \%$ e $14,9 \%$, respectivamente. A tese concluiu que professores de educação física capacitados podem programar um projeto de avaliaçáo do estado nutricional de escolares (AENE) (CEZAR, 2005). O 
projeto AENE funciona no município de Sáo Paulo desde 2005 até os dias atuais.

Merece menção o Projeto Redução dos Riscos de Adoecer e Morrer na Maturidade (RRAMM), desenvolvido na Disciplina de Nutrologia do Departamento de Pediatria da Universidade Federal de São Paulo com o objetivo de implantar um programa interdisciplinar e multiprofissional dirigido à Escola Fundamental para redução dos fatores de risco para a obesidade e as doenças associadas, pela promoção de hábitos saudáveis adequados à realidade da escola pública. Sua proposta de intervenção baseou-se em um programa educativo das áreas de nutrição e atividade física destinado à escola pública, atingindo 2519 crianças matriculadas nas duas primeiras séries do ensino fundamental de oito escolas do município de Sáo Paulo, por meio de treinamento em nutrição e atividade física destinado aos professores (TADDEI, 2002). Deste projeto, que surgiu em 1999, foram derivadas nove dissertaçóes de mestrado nas áreas de nutrição, psicologia e educação física; uma tese de doutorado em nutrição e uma tese de doutorado em pediatria.

Também é interessante destacar o Programa Agita São Paulo, coordenado pela Secretaria de Estado da Saúde de São Paulo (SES), em convênio com o Centro de Estudos do Laboratório de Aptidão Física de São Caetano do Sul (CELAFISCS) e outras instituições parceiras (CEZAR, 2005). O Programa tem como objetivos principais o aumento do nível de atividade física e do nível de conhecimento sobre os benefícios de um estilo ativo de vida saudável, com três focos: escolares, trabalhadores e idosos. Para a intervenção no ambiente escolar foi criado o Agita Galera, Dia da Comunidade Ativa, que envolve mais de 6 milhóes de escolares das 6 mil escolas do estado, localizadas nos 645 municípios. A celebração tem sido realizada a cada última sexta-feira de agosto, desde 1997, sendo que, a partir de 2005, alcançou também as 1000 escolas da capital de São Paulo, acrescentando, assim, mais de um milhão de escolares. $\mathrm{O}$ impacto positivo do Agita São Paulo levou ao reconhecimento da Organização Mundial da Saúde e à consequente constituição da rede mundial Agita Mundo Network (OLIVEIRA, 2006).
Seguindo a linha de intervenção em educação nutricional nas escolas, menciona-se o Programa de Educação Alimentar realizado durante o ano de 2005 com os alunos de uma escola municipal de ensino fundamental do município de Ribeirão Preto/SP. O estudo objetivou desenvolver um programa de educação alimentar e avaliar seus efeitos sobre o estado nutricional, o nível de conhecimento em alimentação e nutrição e as práticas alimentares, além de determinar o nível de atividade física dos escolares. O programa teve duração de seis meses e envolveu 951 alunos, de 6 a 16 anos de idade, matriculados da pré-escola à $8^{\mathrm{a}}$ série, os quais receberam aulas semanais sobre alimentação, nutrição e atividades físicas. Após a intervenção, o estudo concluiu que o programa de educaçáo alimentar proposto foi eficaz para melhorar o nível de conhecimento sobre alimentação, nutrição e práticas alimentares, no entanto, tais melhoras não foram capazes de modificar positivamente o estado nutricional dos alunos. Além disso, foi detectada baixa aderência a programas de atividades físicas (SCHMITZ et al, 2008).

Outro parâmetro relacionado à saúde que tem suscitado projetos de intervenção nas escolas é o nível de atividade física dos alunos. Vemos, pois, que educação nutricional e educação física são áreas que predominam entre os projetos de saúde escolar. Neste sentido, chama atenção uma dissertaçáo de mestrado apresentada no Programa de Pós-Graduação stricto sensu em Educação Física da Universidade Católica de Brasília. $\mathrm{O}$ estudo foi desenvolvido em escolas do ensino fundamental das cidades satélites de Samambaia e Riacho Fundo II, também no Distrito Federal, e teve como objetivo verificar o efeito de uma intervenção de caráter educativo na promoção de um estilo de vida saudável. Foram avaliados o nível de atividade física e os fatores de risco para a saúde: obesidade e hipertensão arterial. A intervenção foi realizada na escola experimental em diversos procedimentos, como: palestra com alunos e pais, entrega de panfletos, folders e guias de bolso, interferência nas aulas de educação física e nas atividades denominadas: recreio ativo e 'sabadão' da saúde. Todos os procedimentos deram ênfase à importância de manter um estilo de vida ativo e, consequentemente, saudável, não só na escola, mas também no cotidiano. 
O estudo encontrou elevados índices de sedentarismo, sobrepeso e obesidade nas escolas, e pôde constatar uma diminuição do sedentarismo nos estudantes da escola experimental após a intervenção, ratificando, dessa forma, a importância de projetos dessa natureza nas escolas (CAVALCANTI, 2009).

De forma similar, o estudo de Ribeiro e Floriano (2010) avaliou os efeitos de um programa de intervenção no nível de atividade física de adolescentes de escolas públicas de uma regiáo de baixo nível socioeconômico da cidade de São Paulo/SP. A amostra final foi constituída por 69 adolescentes, de 12 a 14 anos, alocados em dois grupos de intervenção (educação em atividade física e saúde e esportes/exercícios físicos) e em um grupo controle. A intervenção do grupo educação em atividade física e saúde foi planejada para afetar os padrôes de atividade física e os hábitos alimentares adotados na adolescência. As estratégias utilizadas com esse grupo incluíram discussóes, debates, dinâmicas de grupo e sessōes de atividades físicas. A intervenção do grupo esportes/exercícios físicos incluiu o desenvolvimento dos fundamentos de quatro modalidades esportivas e a participação em jogos pré-desportivos para elevar o nível de atividade física dos participantes. No grupo controle, foram realizados dois encontros, com duração de 50 minutos cada, para garantir o acesso às mesmas informações transmitidas ao grupo educação em atividade física e saúde, de maneira resumida. Foi utilizado um questionário que avalia a prática da atividade física semanal e anual, no interstício de agosto a dezembro de 2008. Concluiu-se que, apesar das poucas sessôes de atividades físicas, o programa proposto para o grupo educação em atividade física e saúde foi bem aceito pelos adolescentes, reforçando a hipótese de que a escola é o local ideal para promover atividade física e saúde em escolares adolescentes.

Registrou-se cerca de 600 programas denominados Saúde na Escola, incentivados pelo governo e implementados por escolas públicas e particulares com o objetivo de desenvolver açóes de promoção da saúde e prevenção de agravos que permitam, entre outros fatores, melhoria do rendimento escolar, recuperação da autoestima e da autoconfiança e diminuição dos níveis de absenteísmo e repetência escolar. Em 2008, os
Ministérios da Saúde e da Educação lançaram o Programa Saúde na Escola (PSE) com os objetivos de reforçar a prevenção à saúde dos alunos brasileiros e construir uma cultura de paz nas escolas. O programa envolve diversos setores (municípios, órgãos federais, ministérios, Programa Saúde da Família, Universidade Aberta do Brasil e outros) e está estruturado em quatro blocos: avaliação das condições de saúde; promoção da saúde e da prevenção; educação permanente e capacitação de profissionais e de jovens; avaliação da saúde dos estudantes por intermédio de duas pesquisas em parceria com o Instituto Brasileiro de Geografia e Estatística (BRASIL, 2008).

Gabriel, Santos e Vasconcelos (2008) avaliaram um programa para promoção de hábitos alimentares saudáveis em escolares do ensino fundamental de Florianópolis (SC). O estudo de intervenção foi desenvolvido com 162 escolares de terceira e quarta séries de duas instituiçốes de ensino, uma pública e outra privada. A metodologia envolveu a aplicação de um questionário de consumo alimentar e o aferimento de peso, estatura, idade e sexo, antes e após um mês de finalizada a intervençấo, de acordo com os protocolos recomendados pela Organização Mundial de Saúde para a coleta de medidas antropométricas. Ao final do programa, não foram constatadas alterações significativas no perfil nutricional dos escolares, entretanto, é preciso considerar que o tempo entre a conclusão do programa educativo e a realização do segundo exame antropométrico (aproximadamente um mês) pode não ter sido suficiente. Porém, houve redução significativa do consumo de bolachas recheadas pelos meninos da escola particular e aumento do consumo de merenda escolar e frutas na escola pública, ou seja, houve mudanças de atitudes.

Também é interessante o trabalho de Zapater $\mathrm{et} \mathrm{al}$, (2004), que aplicaram um programa de educação postural em escolares de Bauru,/SP, e, apesar de realizarem apenas uma sessão educativa, contaram com reforço de professores. Tal como no presente estudo, verificaram boa aceitação por parte dos escolares, uma vez que o programa proporcionou aumento do conhecimento da postura sentada adequada, mas não podem afirmar se houve mudança de hábitos, e sugerem acompanhamento por maior tempo. 


\section{Discussão}

O enfoque dos programas de intervençáo em saúde realizados nas escolas brasileiras está relacionado, na sua ampla maioria, a dois aspectos: prática de atividade física e alimentaçáo saudável. Especificamente sobre atividade física, parece haver uma relação direta de causa-efeito entre essa variável e a melhoria da saúde (CARMO JÚNIOR, 2009). Recentemente, a Pesquisa Nacional de Saúde do Escolar (PeNSE) (IBGE, 2009), que entrevistou mais de 62 mil adolescentes de todas as capitais do Brasil, mostrou que apenas $43,1 \%$ dos adolescentes atingiram a recomendação de prática de atividade física, sendo maior a participação entre os escolares do sexo masculino e aqueles matriculados na rede privada de ensino. Informaçóes acerca dos comportamentos sedentários são ainda mais escassas, e os poucos dados existentes apontam para prevalências elevadas de jovens despendendo um tempo excessivo em atividades sedentárias (MOLINA et al, 2010; TASSITANO et al, 2010), com quase $80 \%$ dos adolescentes brasileiros relatando assistir duas ou mais horas de TV por dia (IBGE, 2009).

As pessoas estão consumindo mais alimentos de grande densidade energética, com altos teores de açúcar e gorduras saturadas, ou excessivamente salgados. Essa forma de nutrição, aliada a um sedentarismo crescente, está ocorrendo muito mais rapidamente nos países em desenvolvimento do que nos desenvolvidos. Consequentemente, as doenças crônicas são cada vez mais comuns nos países em desenvolvimento, especialmente nos mais pobres. Esse fato implica um encargo duplo, uma vez que aos casos crônicos se somam as doenças infecciosas que continuam a assolar os países menos favorecidos do ponto de vista socioeconômico (ORGANIZAÇÃO PAN-AMERICANA DA SAÚDE, 2003).

$\mathrm{O}$ ambiente físico escolar tem sido alvo de pesquisas recentes. Esta abordagem discute as políticas escolares com relação aos aspectos de oportunidade de equipamentos, espaços fiscos adequados, qualidade das aulas de educação física, tipo de construção e comportamento sedentário durante o período escolar. Mesmo sendo um campo ainda carente de consistência em seus instrumentos, os resultados săo muito interessantes no que diz respeito à formulaçáo de intervençôes específicas dentro do ambiente escolar. Pré-escolas e escolas de ensino fundamental e médio, cujos ambientes físicos são mais adequados, têm alunos mais ativos (DOWDA et al, 2009).

As instituiçóes de saúde, a família e o ambiente escolar sáo recursos importantes, que podem influenciar de forma positiva ou negativa o compromisso e o envolvimento das pessoas com comportamentos que levam à promoção da saúde. Dessa forma, podem ser vistas como benefícios ou barreiras para a aquisiçấo de hábitos saudáveis (GUEDES et al, 2009).

Muitos projetos e programas realizados na comunidade escolar tiveram a participação efetiva dos professores de educação física. Em suma, os professores de educaçáo física podem contribuir sobremaneira para a educação e a promoção da saúde. Ao utilizar os dados antropométricos e outras mensuraçóes, podem detectar a prevalência das enfermidades hipocinéticas e, a partir daí, orientar os alunos nas aulas de educaçáo física sobre a prática regular de atividades físicas, assim como sobre outras noçōes básicas de saúde. Neste sentido, a educaçáo física escolar é a disciplina que reúne as melhores condiçóes para proposiçāo de estratégias de intervenção.

É importante destacar que o Ministério da Saúde, com o propósito de incentivar e fortalecer a avaliaçáo dos projetos de intervenção em atividade física financiados pelo governo federal, formalizou e consolidou parcerias com instituiçóes de ensino e pesquisa nacionais e internacionais, como: Universidade Federal de Minas Gerais (UFMG); Universidade Federal de Sergipe (UFS); Universidade Federal de Pelotas (UFPel); Universidade de São Paulo (USP); Universidade Federal de São Paulo (Unifesp); Saint Louis University; Centers for Disease Control and Prevention dos Estados Unidos da América (CDC/USA); e a Organização Pan-Americana da Saúde (OPAS-OMS) (BRASIL, 2011).

$\mathrm{O}$ resultado deste trabalho produziu uma revisão de literatura científica do Brasil e da América Latina, que identificou os projetos de intervençáo que apresentaram evidências de efetividade no campo da atividade física/práticas corporais. A revisão pôde verificar a existência de poucos programas de intervençáo em atividade física nas escolas e nos núcleos de apoio à saúde da 
família (NASF), com reduzida participação dos profissionais de educação física nas equipes, e inferir o quanto é importante disseminar, entre gestores e trabalhadores do SUS, a prática de programas e açóes para a promoção da saúde com ênfase na valorização do trabalho do profissional de educação física (BRASIL, 2011). Mais uma conclusão importante deste trabalho de revisão de literatura, a partir da análise dos estudos de intervenção publicados na América Latina e no Brasil, em particular, foi de que os programas que promovem atividade física na escola foram bem-sucedidos na redução do sedentarismo (MALTA et al, 2009).

Outra questão importante, quando se aborda programas de intervenção em saúde no ambiente escolar, diz respeito ao controle e à prevenção do sobrepeso e da obesidade, devido ao aumento dos índices internacionais da obesidade infanto-juvenil nas últimas décadas. Em vista disso, a Organização Mundial de Saúde publicou, em 2004, o documento "Estratégia Global para Nutrição, Atividade Física e Saúde", no qual sugere, no tocante à alimentação, maior ingestão de verduras, legumes e frutas e menor ingestão de sal, gorduras saturadas e açucares; quanto à atividade física, propóe o aumento do nível de atividade física das crianças na escola, com especial atenção ao transporte ativo, em bicicletas ou a pé (WORLD HEALTH ORGANIZATION, 2004). No Brasil, esse tema proporcionou o desenvolvimento de diversos projetos e programas de educação relacionados à alimentação e à nutrição na escola.

Como adendo, deve-se dizer que a presente revisão possui limitaçóes. A primeira refere-se ao fato de que pesquisas bibliográficas realizadas na internet têm um elevado grau de complexidade devido à amplitude das redes de informação, o que pode ter ocasionado a não citação de programas e/ou projetos de intervenção escolar. A segunda refere-se a não inclusão de investigações sobre prevalências de doenças crônicas não transmissíveis, o que, por sua vez, não deixa de ser uma intervenção na escola. No entanto, a inclusão de todas essas informaçóes elevaria a dimensão do texto, tornando-o incompatível como o formato da revista. Por outro lado, a vantagem deste estudo é mostrar que a escola, através da Educação Física, pode ser o espaço ideal para promover saúde entre os escolares, através de intervençóes educativas no período de maior declínio dos padróes de atividade física e dos hábitos alimentares.

\section{Conclusões}

O crescimento econômico e populacional está acarretando mudanças no estilo de vida e nos hábitos alimentares da população brasileira, que influenciam o desenvolvimento das doenças crônicas não transmissíveis. Diante desse fato, no Brasil, o poder público está incentivando e financiando projetos de educação e promoção da saúde nas escolas, através da Política Nacional de Promoção da Saúde (PNPS). A ideia é abandonar a linha assistencialista complementar e partir para um conjunto integrado de açóes de educação em saúde que se inicia na escola e estende-se à família.

$\mathrm{Na}$ teoria, os projetos deveriam envolver os diversos profissionais de saúde, que, em conjunto com professores e direção das escolas, devem ser agentes multiplicadores de informaçôes sobre diversos assuntos relacionados à saúde, não somente a alunos, mas também aos seus familiares, permitindo, assim, que comunidades possam adotar comportamentos e estilos de vida mais saudáveis. No entanto, os programas de intervenção em saúde não ocorrem na maioria das escolas brasileiras, e os que acontecem geralmente são promovidos por instituiçóes científicas e serviços de saúde. Neste sentido, a escola, como instituição formadora da juventude, tem um papel estratégico no desenvolvimento de açóes e na aplicação de programas educacionais capazes de melhorar as condições de saúde, desde que possua um enfoque crítico, participativo, interdisciplinar, transversal e que consistam em processos lúdicos e interativos.

As açóes de saúde mais identificadas nos programas e projetos avaliados referem-se ao incentivo da prática de atividades físicas e da alimentação saudável. Neste sentido, os programas que promoveram atividade física na escola foram bem-sucedidos na redução do sedentarismo. Nos núcleos de apoio à saúde da família (NASF), verificou-se reduzida participação dos profissionais de educação física nas equipes. Adicionalmente, deve-se considerar o potencial do Profissional da 
Educação Física a partir do momento em que o mesmo deve cumprir com uma das suas principais atribuições, que é proporcionar conhecimentos teóricos e práticos sobre aptidão física e saúde, a fim de que os indivíduos possam adotar hábitos saudáveis não só na escola, mas em suas vidas.

\section{Referências}

BRASIL. Ministério da Saúde. Saúde na Escola. Brasília, 2008. Disponível em: <http://portalsaude.saude.gov.br/portalsaude/ texto/1752/355/saude-na-escola.html>. Acesso em: 20 ago. 2010.

Ministério da Saúde. Secretaria de Vigilância em Saúde. Avaliação de Efetividade de Programas de Atividade Física no Brasil. Brasília: Ministério da Saúde, 2011. 184 p.

Ministério da Saúde. Vigilância de fatores de risco e proteção para doenças crônicas por inquérito telefônico. Brasília, DF, 2010. 151p. Disponível em: <http://portal.saude.gov.br/portal/ arquivos/pdf/vigitel2010_final_web.pdf>. Acesso em: 10 mar. 2012. 2009. 44 p.

Redes de produção de saúde. Brasília: Ministério da saúde,

CARMO JÚNIOR, T. R. A intervenção escolar como meio de promover o estilo de vida ativo em escolares de 7 a 11 anos. 2009. 84 f. Dissertação (Mestrado em Educação Física) - Universidade Católica de Brasília, Brasília, 2009.

CAVALCANTI, L. A. Efeitos de uma intervenção em escolares do ensino fundamental I, para a promoção de hábitos alimentares saudáveis. 2009. 92 f. Dissertação (Mestrado em Educação Física) Universidade Católica de Brasília, Brasília, 2009.

CEZAR, C. Avaliação do estado de nutrição de escolares do município de São Paulo: uma experiência multidisciplinar envolvendo professores de educação física do ensino fundamental e médio. 2005. 128 f. Tese (Doutorado em Nutrição Humana Aplicada) Universidade de São Paulo, São Paulo, 2005.

DOWDA, M. et al. Policies and characteristics of the preschool environment and physical activity of young children. Pediatrics, Elk Grove Village, IL, v. 123, n. 2, p. 261-266, 2009.

GABRIEL, C. G.; SANTOS, M. V.; VASCONCELOS, F. A. G. Avaliação de um programa para promoção de hábitos alimentares saudáveis em escolares de Florianópolis, Santa Catarina, Brasil. Revista Brasileira de Saúde Materno Infantil, Recife, v. 8, n. 3, p.299-308, jul. 2008.

GONÇALVES, F. D. et al. Health promotion in primary school. Interface - Comunicação, Saúde e Educação, Botucatu, São Paulo, v. 12, n. 24, p. 181-92, jan./mar. 2008.
GUEDES, N. G et al. Atividade física de escolares: análise segundo o modelo teórico de promoção da saúde de Pender. Revista da Escola de Enfermagem - USP, São Paulo, v. 43, n. 4, p. 774-780, 2009.

INSTITUTO BRASILEIRO DE GEOGRAFIA E ESTATÍ́STICA (IBGE). Pesquisa Nacional de Saúde do Escolar. Rio de Janeiro: Ministério da Saúde, 2009.

MALTA, D. C. et al. A política nacional de promoção da saúde e a agenda da atividade física no contexto do SUS. Epidemiologia e serviço de saúde, Brasília, v. 18, n. 1, p. 79-86, mar. 2009.

MOLINA, M. C. B. et al. Fatores de risco cardiovascular em crianças de 7 a 10 anos de área urbana, Vitória, Espírito Santo, Brasil. Cadernos de Saúde Pública, Rio de Janeiro, v. 26, n. 5, p. 909-917, 2010.

MOURA, G.C. et al. Association between physical activity and quality of life in adults. Revista de Saúde Pública, São Paulo, v. 46, n. 1, p. 1-12, 2012.

OLIVEIRA, L. C. Avaliação de efetividade de uma intervenção em promoção da atividade física e da saúde no ambiente escolar. 2006. 94 f. Dissertação (Mestrado em Educação Física) - Universidade São Judas Tadeu, São Paulo, 2006.

ORGANIZAÇÃO PAN-AMERICANA DA SAÚDE (OPAS). Doenças crônico-degenerativas e obesidade: estratégia mundial sobre alimentação saudável, atividade física e saúde. Brasília: Organização Pan-Americana da Saúde, 2003. 60 p.

PIRES, C.G.S.; MUSSI, F. C. Crenças em saúde para o controle da hipertensão arterial. Ciência e Saúde Coletiva, Rio de Janeiro, v. 13, supl. 2, p. 2257-2267, 2008.

POZENA, R.; CUNHA, N. F. S. Projeto Construindo um futuro saudável através da prática da atividade física diária. Saúde e Sociedade, São Paulo, v. 18, supl. 1, p. 52-56, 2009.

REIS, M.S.; REIS, R.S.; HALLAL, P.C. Validity and reliability of a physical activity social support assessment scale. Revista de Saúde Pública, São Paulo, v. 45, n. 2, p. 1-8, 2011.

RIBEIRO, E. H. C.; FLORINDO, A. A. Efeitos de um programa de intervenção no nível de atividade física de adolescentes de escolas públicas de uma região de baixo nível socioeconômico: 
descrição dos métodos utilizados. Revista Brasileira de Atividade Física e Saúde, Florianópolis, v. 15, n. 1, p. 28-34, 2010.

SANTOS, F. P. A. et al. Estratégias de enfrentamento dos dilemas bioéticos gerados pela violência na escola. Revista de Saúde Coletiva, Rio de Janeiro, v. 21, n.1, p. 267-281, 2011.

SANTOS, Z. M. S. A.; CAETANO, J. A.; MOREIRA, F. G. A. Atuação dos pais na prevenção da hipertensão arterial: uma tecnologia educativa em saúde. Ciência e Saúde Coletiva, Rio de Janeiro, v. 16, n. 11, p. 4385-4394, 2011.

SCHMITZ, B. A. S. et al. A escola promovendo hábitos alimentares saudáveis: uma proposta metodológica de capacitação para educadores e donos de cantina escolar. Cadernos de Saúde Pública, Rio de Janeiro, v. 24, supl. 2, p.S312-S322, 2008.

TADDEI, J. A. A.C. Redução dos riscos de adoecer e morrer na maturidade: Projeto RRAMM. São Paulo: Universidade Federal de São Paulo, 2002. Disponível em: <http://www.unifesp.br/dped/ disciplinas/nutricao/projet.html> Acesso em: 23 set. 2010.

TASSITANO, R.M. et al. R.S. Enrollment in physical education is associated with health-related behavior among high school students. Journal of School Health, Bethesda, v. 80, n. 3, p. 126-133, mar. 2010.
TENDAIS, I. et al. Physical activity, health-related quality of life and depression during pregnancy. Cadernos de Saúde Pública, Rio de Janeiro, v. 27, n. 2, p. 219-228, fev. 2011.

VERGNAUD, A.C. et al Weight fluctuations and risk for metabolic syndrome in an adult cohort. International Journal of Obesity, London, v. 32, n. 2, p. 315-21, 2008.

WORLD HEALTH ORGANIZATION (WHO). Obesity: preventing and managing the global epidemic. Geneva: World Health Organization, 2000. p. i-xii, 1-253p. (WHO Technical Report Series, 894).

WORLD HEALTH ORGANIZATION (WHO). Global Strategy on diet, physical activity and health. Geneva: World Health Organization, 2004. 21 p.

ZAPATER, A. R.; SILVEIRA, D. M.; VITTA, A.; PADOVANI, C. R.; SILVA, J. C. P. Postura sentada: a eficácia de um programa de educação para escolares. Ciência e Saúde Coletiva, Rio de Janeiro, v. 9, n. 1, p. 191-199, 2004.

Recebido para publicação em Maio/2012

Versão definitiva em Outubro/2012

Suporte financeiro: não houve

Conflito de interesse: inexistente 